Zeitschrift für Wissenschaftliche und technische Kolloidchemie
Organ für das Gesamtgebiet der reinen und angewandten Kolloidchemie
Priv.-Doz. Dr. Wolfgang Ostwald in Leipzig, Brandvorwerkstraße 77

\title{
Ueber die elektrische Leitfähigkeit von kolloiden Lösungen.
}

\author{
Von Harald Nordenson (Upsala).

Die Frage nach der Leitfähigkeit der Kolloide ist Gegenstand einer großen Zahl von Untersuchungen gewesen ${ }^{1}$ ).

$\mathrm{DaB}$ die Kolloide eine gewisse „Eigenleitfähigkeit" besitzen müssen, geht schon daraus hervor, dab die Teilchen elektrisch geladen sind und durch den Strom bewegt werden, und also zu einem gewissen Betrag den Elektrizitätstransport mit besorgen. Ueber die Größe dieser Eigenleitfähigkeit liegen aber sehr verschiedene experimentelle Resultate vor.

J. Ducla ux, der eine Reihe verschiedener Kolloide, besonders nichtmetallischer wie Eisenoxyd und Ferrocyanverbindungen untersuchte, hat stets sehr beträchtliche Werte der Leitfähigkeit gefunden ${ }^{2}$ ). Bei metallischen Kolloiden sind Messungen u. a. von E. F.Burton ${ }^{3}$ ), W. R. Whitney und J. C. Blake ${ }^{4}$ ) ausgeführt worden. Letztere geben für eine mit Azetylen hergestellte Goldlösung den Wert $\varkappa_{18}=12,7 \cdot 10^{-6}$, für ein Bredig'sches Platinsol $x_{18}=2,9 \cdot 10^{-6}$. E. F. Burton gibt dagegen für ein Bredigsches Sol von Silber den Wert $x_{18}=31,0.10^{-6}$, und von Gold $x_{18}=3,6 \cdot 10^{-6}$.

Wie aber $H$. Freundlich richtig hervorhebt $^{5}$ ), muß darauf Rücksicht genommen werden, daß auch bei sehr ausgiebiger Dialyse - die oben erwähnten Lösungen waren alle gut dia-

1) Vgl. Wo. Ostw a ld, Grundriß der Kolloidchemie (1909), 243.

2) Koll.- Zeitschr. 3, 126 (1908); Journ. de Chimie phys. 7, 405 (1909).

3) E. F. Burton, Phil. Mag. [6] 12, 475 (1906).

4) W. R. Whitn e y u. J. C.B lake, Journ. Amer. Chem. Soc. 26, 1339 (1904).

5) H. Freundi i ch, Kapillarchemie (Leipzig 1909), 337. lysiert - immer kleine Mengen von Elektrolyten durch Adsorption an dem Kolloid haften und bei der Messung die Resultate stark beeinflussen können.

Die von $H$. Freundlich aufgeworfene, bis jetzt offene Frage, in welchem Maße die Elekirolyte einerseits und das Kolloid allein anderseits Ursache der Leitfähigkeit sind, kann aber durch folgende Auseinandersetzungen näher beleuchtet werden.

Wenn eine gewisse Menge eines Elektrolyts z. B. eines Metallsalzes kristalloid aufgelöst ist und vollständige Dissoziation vorausgesctht wird, so setzt sich die Leitfähigkeit der Lösung aus der der Kationen und der der Anionen additiv zusammen. Findet nun eine Umwandlung (z. B. Reduktion) statt, so daf das Metall in kolloider Form ausgefällt wird, und sämtliche freien Elektrolyte als weggetrieben gedacht werden, so wird die Leitfähigkeit der Lösung vom Kolloid allein d. h. teils von den geladenen Metallpartikeln, teils von den von ihnen abdissoziierten Ionen herrühren.

Nehmen wir nun an, daß die Wanderung der Teilchen sowie der lonen dem Stokesschen Gesetz gehorcht --- was wenigstens sehr nahe richtig ist ${ }^{6}$ ) - und bezeichnen wir den kolloiden Zustand mit k, den ionisierten mit i, so ergibt sich

$$
\mathrm{u}_{\mathrm{i}}=\frac{\mathrm{H} \mathrm{e}_{\mathrm{i}}}{6 \pi \eta \mathrm{r}_{\mathrm{i}}} ; \quad \mathrm{u}_{\mathrm{k}}=\frac{\mathrm{H} \mathrm{e}_{\mathrm{k}}}{6 \pi \eta \mathrm{r}_{\mathrm{k}}} ;
$$

wo $\mathbf{u}$ die Wanderungsgeschwindigkeit, $\mathrm{H}$ den

6) Mehrere Untersuchungen haben die Gültigkeit dieses Gesetzes für die Kolloide experimentell bestätigt. Vgl. J. Nordlund, Ark. f. Mat. Fys. usw. 9, 13 (1911); A. We stgren, Zeitschr. f. physik. Chemie 89,63 (1914). 
Potentialfall pro $\mathrm{cm}, \eta$ die Viskosität des Mittels, e die Ladung und $\mathrm{r}$ den Radius der Partikeln bezeichnen.

Sogar alle Untersuchungen über die Wanderungsgeschwindigkeit suspendierter und kolloider Partikeln haben nun gelehrt, daß diese von derselben Größenordnung wie die der Ionen (mit Ausnahme von $\mathrm{H}^{\cdot}$ und $\mathrm{OH}^{\prime}$ ) ist, und daB sie sich bei wachsender Ladung der Teilchen den Werten der Ionen als obere Grenze nähert ${ }^{7}$ ). Man kann also in den obenstehenden Formeln $u_{k}=u_{i}$ setzen $^{8}$ ), und erhält dadurch

$$
\begin{aligned}
\frac{e_{k}}{e_{i}} & =\frac{r_{k}}{r_{i}} ; \\
\text { oder } \frac{e_{k}}{r_{k}} & =e_{i} \\
r_{i} & =\text { konst. }
\end{aligned}
$$

das heißt, die maximale elektrische Ladung eines kolloidenoder suspendierten Partikels ist seinem Radius proportional ${ }^{9}$ ).

Die von der gelösten Substanz hervorgerufenen Leitfähigkeiten $l_{i}, l_{k}$ in den beiden Lösungen - - wo die Substanz einerseits iondispers, anderseits kolloid gelöst ist - sind aber sowohl der Ladung der Partikeln bzw. Ionen als auch ihrer Zahl (n) proportional, und man erhält also

$$
\frac{l_{k}}{l_{i}}=\frac{n_{k} e_{k}}{n_{i} e_{i}}
$$

Nun wurde aber in beiden Fällen dieselbe Gewichtsmenge Substanz angenommen, und die Partikel- und Ionenzahlen verhalten sich also umgekehrt wie die dritten Potenzen der Radien

woraus sich

$$
\frac{\mathrm{n}_{\mathrm{k}}}{\mathrm{n}_{\mathrm{i}}}=\left(\frac{\mathrm{r}_{\mathrm{i}}}{\mathrm{r}_{\mathrm{k}}}\right)^{3}
$$

$$
\frac{l_{k}}{l_{i}}=\left(\frac{r_{i}}{r_{k}}\right)^{3} \cdot \frac{r_{k}}{r_{i}}
$$

und

ergibt.

$$
\frac{1_{k}}{l_{i}}=\left(\frac{r_{i}}{r_{k}}\right)^{2}
$$

Die von einer gewissen Substanzmenge hervorgerufenen Leitfähig-

7) Vgl. H. Freund lich, Kapillarchemie (1909), 340; R. Zsigm ondy, Kolloidchemie (1912) 46, 47.

8) Eigentlich $u_{k} \leq u_{i}$

9) Diese Gesetzmäßigkeit ist eine allgemein gültige, also auch für lonen verschiedener Ladung. Wir finden also, daß dasselbe Ion bei verschiedener Wertigkeit einen anderen Radius haben mul3. Dies wird durch die Annahme eines Zuwachses des Ions durch Hydratation erklärt. Vgl. E. W. W a shburn, Jahrb. d. Radioaktiv. 6, 69 (1909); G. v. Hevesy, Jahrb. d. Radioaktiv. 11, 419 (1914). keiten, wenn sie einerseits kolloid, anderseits iondispers aufgelöst wird, verhalten sich also umgekehrt wie die $z$ weite Potenz der Radien.

Es ist somit möglich, wenn die Konzentration und die feilchengröße e iner kolloiden Lösung bekannt sind, ihre Leitfähigkeit oder wenigstens die Größenordnung derselben $z u$ berechnen und damit festzustellen, ob sie auch meBbare Werte erreicht.

Nehmen wir z. B. an, daß wir es mit einer gewöhnlichen kolloiden Silberlösung zu tun haben mit einem Teilchenradius von etwa $25 \mu \mu$, und führen wir als Wert des Radius der Silbermoleküle $0,25 \mu \mu$ ein (in Uebereinstimmung mit den aus der kinetischen Gastheorie und einigermaßen mit den aus Diffusionsmessungen berechneten Werten) ${ }^{10}$ ), so verhalten sich die $\mathrm{Ra-}$ dien wie $1: 100$, und unsere Formel gibt die Relation

$$
l_{k}=\frac{l_{i}}{10^{4}}
$$

Da nun die molekulare Leitfähigkeit des Silbers bei großer Verdünnung den Wert $55\left(18^{\circ}\right)$ erreicht, erhalten wir, wenn die Konzentration des Silbers in Normalitäten mit $\mathrm{c}$ bezeichnet wird

$$
\begin{aligned}
& \mathrm{l}_{\mathrm{i}}=55 \cdot \mathrm{c} \cdot 10^{-3} ; \\
& \mathrm{l}_{\mathrm{k}}=55 \cdot \mathrm{c} \cdot 10^{-7}
\end{aligned}
$$

Die totale Leitfähigkeit des Kolloids setzt sich, wie die des Elektrolyts, aus der der Partikeln und der der abdissoziierten Ionen additiv zusammen. Nehmen wir an, daß die abdissoziierten Ionen Wasserstoffionen sind (günstigster Fall) und bezeichnen wir die Leitfähigkeit des Kolloids $\left(18^{\circ}\right)$ mit $x_{k}$, so erhalten wir

$$
x_{\mathrm{k}}=(314+55) \text { c } 10^{-7}
$$

Es geht aus dieser Formel hervor, daß c sehr große Werte annehmen muß, um meßbare Werte von $x_{\mathrm{k}} \mathrm{zu}$ erzielen. Im allgemeinen ist aber c bei kolloiden Metallösungen sehr klein, schon die Konz. $1 \mathrm{Millimol} / \mathrm{Lit}$. ist eine ziemlich große, und auch dieser Wert für c gibt für das Kolloid in Frage nur $\varkappa_{\mathrm{k}}=3,7 \cdot 10^{-8} \mathrm{~d}$.h. einen nicht me $B$ bareren Wert der Leitfähigkeit. Die Leitfähigkeit der Lösung wird nurvon den anwesenden freien Elektrolyten bestim mt.

Dies wird nun auch für kolloide Metalle ganz allgemein der Fall sein, weil sie fast immer

10) Vgl. The Svedberg, Die Existenz der Molekïle (1912), 63 . 
wenig konzentriert und mäßig hoch dispers sind. Dazu kommt noch, daß die Approximationen hier alle zu Gunsten eines höheren Wertes von $\%_{\mathrm{k}}$ gemacht worden sind. So erreicht $\mathrm{u}_{\mathrm{k}}$ oft nur etwa $1 / 2-1 / 3$ der Werte von $u_{i}$ und also werden nach der Formel $2-3 \mathrm{mal} z u$ große Werte von $l_{k}$ erhalten. Auch die Annahme von $\mathrm{H} \cdot$ - Ionen als die vom Kolloid abdissoziierten gibt einen viel höheren Wert als die Annahme von z. B. Ag-Ionen im Ag-Kolloid.

Wir finden also, daß die Eigenleitfähigkeiten der Kolloide so außerordentlich viel kleinere Werte als die der-iondispersen Lösungen besitzen müssen, daß sie sogar in vielen Fällen nicht meßbare Werte erreichen können.

So können wir mit Rücksicht auf das oben Gesagte nun feststellen, daß z. B. die von Burton, Whitney und Blake (vgl. oben) wiedergegebenen Werte der Leitfähigkeit kolloider Metallösungen, welche von mäßiger Konzentration und Teilchengröße waren, in dem Sinne fehlerhaft sind, daB die erhaltenen Werte nicht vondem Kolloid, sondern von den anwesenden Elektrolyten herrühren müssen.

Dies mag auch in bezug auf einige Messungen von A. Scala und M. Traube-Mengarini - in Zusammenhang mit ihren Versuchen über kolloide Auflösung von Metallen, in Wasser ausgeführt ${ }^{11}$ ) - besonders hervorgehoben werden. Destilliertes Wasser wurde in Schalen von Platin, Silber und Porzellan bis auf das halbe Volumen eingekocht, und dabei stieg die ursprüngliche Leitfähigkeit $x=5.10^{-6}$ auf 16 bzw. 36 und $42 \cdot 10^{-6}$, was sie als einen Beweis dafür hervorheben, daB die nach ihrer Hypothese entstehenden Kolloide eine mebbare Leitfähigkeit haben. Abgesehen davon, daß in einigen dieser Versuche ein Kolloid nicht entsteht, zeigen diese Versuche natürlich nur, daß durch Kochen die Elektrolytenmengen und damit auch die Leitfähigkeiten vermehrt wurden. Die Einwände, welche die Verfasser auf Grund dieser Versuche gegen die Methode F. Kohlra usch's, Leitfähigkeitswasser herzustellen, erheben, sind somit ganz unbegründet. Die vielleicht genauesten Messungen der Leitfähigkeit von kolloiden Metallösungen sind von V. Koh l s ch ütter an Silbersolen ausgeführt worden ${ }^{12}$ ). Diese Sole wurden durch Reduktion einer AgOH-Lösung mit Wasserstoffgas hergestellt, und bei den Messungen wurde immer

11) Koll.-Zeitschr. 6, 69 (1910).

12) Zeitschr. f. Elektrochemie 14, 49, (1908). auf den durch Analyse festgestellten Restgehalt an AgOH Rücksicht genommen. Seine Resultate werden in folgender Tabelle und im Kurvenbild 1 wiedergegeben.

\section{Tabelle I.}

Leitfähigkeit von $\mathrm{AgOH}-\mathrm{Lösung}$ und daraus durch Reduktion erhaltenem Ag-Kolloid.

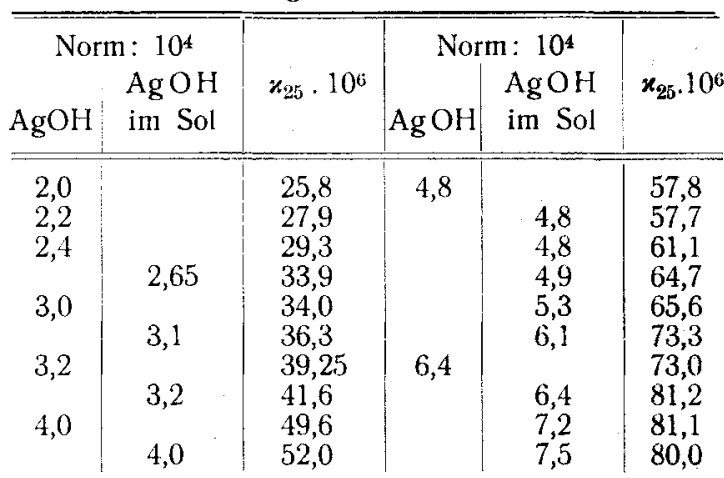

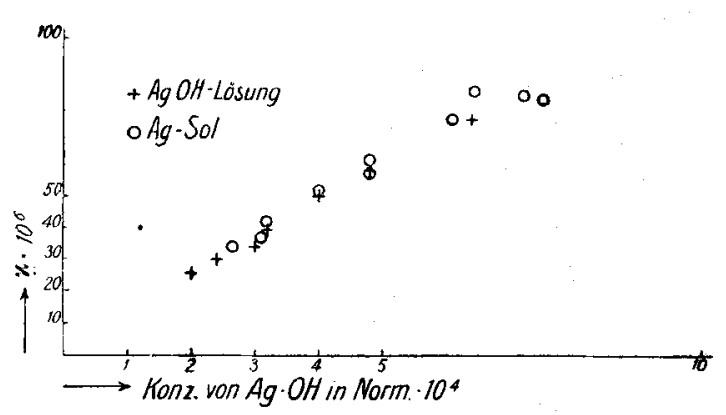

Fig. 1

Es geht daraus hervor, daß die erhaltenen Werte der Gesamtleitfähigkeit der Kolloide innerhalb der Versuchsfehler mit dem aus dem Gehalt an $\mathrm{AgOH}$ berechneten Werte gut übereinstimmen. Da nun die Konzentration seiner Kolloide höchstens etwa $5.10^{-4} \mathrm{Mol} / \mathrm{Lit}$. betrug, geht ja aus dem oben Angeführten hervor, daB die Leitfähigkeit auch keine meßbaren Werte erreichen konnte. Diese Messungen stehen also in gutem Einklang mit den obigen theoretischen Betrachtungen.

Die Untersuchungen der Leitfähigkeit von nicht metallischen Kolloiden, besonders die interessanten und wichtigen Messungen von J. D u cla u x ${ }^{13}$ ), zeigen im Gegensatz zu den obigen Resultaten sehr große Werte der Leitfähigkeit. Diese sehr großen Werte können teils aus den hohen Konzentrationen erklärt werden. Sie

13) J. D $1 \mathrm{c}$ ! a $\mathrm{ux}$, Journ. de Chimie phys. 7, 405 (1909) 
setzen aber auch einen außerordentlich hohen Dispersitätsgrad voraus.

Einige der Werte J. Duclaux's sind in der folgenden Tabelle wiedergegeben, und es geht auch daraus hervor, welche äußerst kleinen Werte des Partikelradius aus scinen Resultaten berechnet werden.

Tabelle II.

\begin{tabular}{l|r|r|r}
\hline & $\begin{array}{c}\text { Konz. in } \\
\text { Proz. }\end{array}$ & $\varkappa .10^{-6}$ & \multicolumn{1}{|c|}{$\begin{array}{c}\text { Radius } \\
\text { in } \mu\end{array}$} \\
\hline Eisenoxyd & 1,84 & 148,5 & 1,95 \\
Berlinerblau & 0,80 & 57,2 & - \\
Thoriumoxyd & 10,30 & 406,0 & 5,20 \\
6,50 & 2418,0 & 0,68
\end{tabular}

Diese Werte der Teilchengröße sind aber gewiß viel zu klein. So berechnet $\mathrm{H}$. B e chhold auf Grund seiner Ultrafiltration der kolloiden Lösungen den Teilchendurchmesser eines gewöhnlichen Eisenoxydsols zu mehr als $50 \mu \mu$. Mit so großen Teilchen würde aber dagegen die Leitfähigkeit der Lösungen in den betreffenden Konzentrationen so hohe Werte nicht erreichen. Die Resultate J. Ducla ux's scheinen also mit meinen Ueberlegungen in Widerspruch zu stehen.

Auch eine andere schwerverständliche Tatsache wurde von J. Duclaux gefunden. Es zeigte sich, daß die Leitfähigkeit der Lösungen nicht der Konzentration proportional zunahm, sondern bei den konzentrierteren Lösungen verhältnismäßig größer war. Um dies zu erklären, nahm J. Duclaux an, daß die Ladung der Teilchen mit der Konzentration zunähme. Experimentell hat er aber gefunden, gerade wie die übrigen Forscher, daß die Wanderungsgeschwindigkeit konstant und von der Konzentration unabhängig war. Wäre aber seine Theorie richtig, würde das $\mathrm{Stokes}$ 'sche Gesetz seine Gültigkeit hier verloren haben. Seine Berechwung der Teilchengröße setzt aber gerade die Gültigkeit dieses Gesetzes voraus. Sogar seine eigenen Berechnungen stehen also mit seiner Theorie im Widerspruch. Da aber, wie oben erwähnt, die strenge Gültigkeit des Stokesschen Gesetzes immer mehr bestätigt worden ist, muB wohl umsomehr die Richtigkeit der Hypothese Duclaux's hinsichtlich der wachsenden Ladung in Frage gestellt werden. Außerdem können diese Schwierigkeiten, wie unten gezeigt wird, auf andere Weise einfacher erklärt werden.

Es sei aber zuerst noch folgendes bemerkt. Die Berechnung der Teilchengröße sowie meine obige Berechnung der Leitfähigkeit setzen voraus, daß3 die Lösungen gleichkörnig sind. Dies ist wohl bei den metallischen Kolloiden ziemlich zutreffend, bei den nichtmetallischen aber sicher nicht. Denn ein derartiges Sol, z. B. von Eisenoxyd, entsteht durch eine nur sehr langsam fortschreitende Hydrolyse und darauffolgende Kondensation des unlöslichen Oxyds. Auch nach ziemlich langer Zeit liegen deswegen Teilchen von den verschiedensten Größen und sogar wohl auch unhydrolysiertes Salz vor. Da nun die Leitfähigkeit mit abnehmendem Radius quadratisch zunimmt, können auch sehr kleine Mengen des Kolloids, wenn sie sich noch im höchstdispersen Zustand befinden, eine verhältnismäßig große Leitfähigkeit erzeugen. Nehmen wir nun auch darauf Rücksicht, daß kleine Mengen von Elektrolyten durch Adsorption am Kolloid haften und sich am Elektrizitätstransport beteiligen können, so werden die großen Werte der Leitfähigkeit leichter verständlich und auch in volle Uebereinstimmung mit meinen theoretischen Betrachtungen gebracht. Die gemessenen Leitfähigkeiten dieser Kolloide rühren alsowahrscheinlich teils von adsorbierten Elektrolyten, teils aber von der Eigenleitfähigkeit des Kolloids her. Letztere rührt ihrerseits von den höchstdispersen Teilen des Kolloids her.

Das Abnehmen der spez. Leitfähigkeit bei verdünnten Lösungen kann folgendermaßen eine Erklärung erhalten. In verschiedenen Verdünnungsgraden geht die Hydrolyse eines Salzes verschieden schnell, und zwar schneller bei gröBerer Verdünnung. Die eventuell adsorbierten Mengen des Ausgangselektrolyts (z. B. $\mathrm{FeCl}_{3}$ ) erleiden deswegen bei Verdünnung einer konzentrierten Lösung eine teilweise Hydrolyse. Dazu kommt noch, daß die Kondensation der ausgeschiedenen unlöslichen Substanz zum Kolloid sehr oft bei Verdünnung beschleunigt wird. Die Verdünnung von konzentrierten Lösungen muß deswegen auch eine Verminderung der spez. Leitfähigkeit herbeiłühren. Nun hebt aber Duclaux selbst hervor, daß seine sämtlichen Versuche mit verschieden konzentrierten L.ösungen so ausgeführt wurden, daß eine stark konzentrierte Ausgangslösung allmählich verdünnt wurde. In sämtlichen Fällen konnte hier also eine Dispersitätsverminderung parallel der Konzentrationsänderung verlaufen und damit, der erwähnten Anschauung gemäß, eine relative Leitfähigkeitsverminderung herbeiführen ${ }^{14}$ ). Diese

14) Vielleicht können auch die Mengen von pro Teilchen adsorbierten Elektrolyten sich mit der Konzentration 
merklichen Abweichungen in den verdünnten Lösungen wären somit einfach auf eine Veränderung des Dispersitätsgrades zurückzuführen.

Zus a m m en assung.

1. Die maximale Ladung eines kolloiden oder suspendierten Partikels ist seinem Radius proportional; $\mathrm{e} / \mathrm{r}$ ist konstant.

2. Wird eine gewisse Substanzmenge einerseits iondispers, anderseits kolloid aufgelöst, so verhalten sich die von ihr hervorgerufenen Leitfähigkeiten umgekehrt wie die zweiten Potenzen der Radien. Demzufolge kann die Eigenleitfähigkeit

des Kolloids verändern. Darüber liegen aber meines Wissens keine experimentellen Ergebnisse in der Literatur vor.

Upsala. eines Kolloids von bekannter Konzentration und bekanntem Dispersitätsgrade berechnet werden.

3. Auf Grund dieser Beziehung ergibtsich, daß die Eigenleitfähigkeiten von mäßig konzentricrten und mäßig hochdispersen Kolloiden - z. B. Metallsolen - nicht meBbare Werte erreichen. Die bei Messung erhaltenen Werte rühren nur von kleinen Mengen von Elektrolyten her.

4. In Kolloiden von sehr hoher Konzentration und besonders von sehr hohem Dispersitätsgrad - also besonders nicht-metallischen Kolloiden kann die Eigenleitfähigkeit dagegen meßbare Werte erreichen. Auch hier muß aber bei den durch Messung erhaltenen Werten dem Einfluß der eventuell adsorbierten Elektrolyte Rechnung getragen werden.

\section{Chemisches Universitätslaboratorium.}

\section{Die Peptisationserscheinungen in Gerbstofflösungen.}

Von W. Moeller (Hamburg).

(Fingrgangen am 12. Februar 1915.)
Ein spezielles Gebiet der Kolloidchemiestellen die sogenannten Peptisationserscheinungen bei Kolloiden dar, welche zuerst von Th. Grah a m ${ }^{1}$ ) beobachtet und genauer untersucht wurden. Grah am fand, daß gewisse Körper in verhältnismäßig geringer Menge in wässeriger Lösung einem Gel zugesetzt, die Fähigkeit besitzen, dieses in ein Sol zu verwandeln. Als Typus dieses Vorganges betrachtete $\mathrm{Graham}$ die Peptisation des Albumins durch das Pepsin, d. h. ein Gemisch kolloider Fermente auf andere Kolloide. Seitdem sind nun sowohl in der organischen als auch anorganischen Chemie zahlreiche Vorgänge bekannt geworden, welche eine völlige Analogie mit der Verdauung des Albumins im tierischen Organismus aufweisen, sodaß die von $\mathrm{Grah}$ a $m$ von diesem Prozeß abgeleitete Bezeichnung der Peptisation eine weitgehende Bedeutung gewonnen hat. Ausführliche Untersuchungen sind von A. Lottermos e ${ }^{2}$ ) und später von B. Szilard ${ }^{3}$ ) bekannt geworden. Neuerdings hat P. P. v. Weimarn $n^{4}$ ) in seinen Untersuchungen über den kolloiden Zustand der Materie die Peptisationserscheinungen berücksichtigt und zu erklären versucht.

1) Th. Grah a m, Ann. d. Phys. 2, 123, 159 (1864).

2) A. Lottermoser, Zeitschr. f. physik. Chem. 60, 451 (1907); 62, 359 (1908).

3) B. Sz il a rd, Beiträge zur allgem. Kolloid-Chem. (Dresden 1908).

4) P. P. v. Weimarn, Zur Lelue von den $Z u-$ ständen der Materie (Dresden 1914.)
Betrachtet man nun die in der Gerberei verwendeten Gerbstofflösungen unter diesem Gesichtspunkte, so findet man, daß sämtliche Lösungen, sowohl die vegetabilischen als auch die mineralischen Gerbmittel, in ihren Eigenschaften eine große Aehnlichkeit mit allen bisher bekannten Peptisationserscheinungen besitzen. Bei näherer Untersuchung auch einer anscheinend noch so einheitlichen Gerbstofflösung kann man feststellen, daß dieselbe mehr oder weniger große Mengen eines Körpers enthält, welcher an sich unlöslich ist in Wasser und nur auf dem Wege der Peptisation durch einen zweiten, chemisch oder auch nur kolloid, löslichen Körper in ein Sol übergeführt wird. Da meines Erachtens die Untersuchung dieser Verhältnisse zur Aufklärung des Gerbphänomens beitragen kann, so werde ich in den folgenden Betrachtungen zunächst den Zustand der Gerbstofflösungen nach der Peptisationstheorie zu erklären versuchen und ferner die sich hieraus für die Gerbtheorie ergebenden Schlüsse ziehen.

Die Folgerungen, zu welchen ich bei meinen Untersuchungen gelangte, lassen sich nun in verschiedene Grundsätze zusammenfassen, welche ich zunächst voranstelle:

I. Eine wahre Lösung kann niemals gerbende Eigenschaften zeigen, sondern nur peptisierte Sole. Alle gerbenden Substanzen cnthalten demnach Gele, welche durch Einwirkung eines lös- 